\title{
Correction to: Antidiabetic and Antioxidant Activity of Green Synthesized Starch Nanoparticles: An In Vitro Study
}

\author{
Vishnupriyan Varadharaj ${ }^{1} \cdot$ Arulvel Ramaswamy $^{2} \cdot$ Rajeswari Sakthivel $^{1} \cdot$ Ramasamy Subbaiya $^{2}$. \\ Hamed Barabadi ${ }^{3} \cdot$ Murugesan Chandrasekaran $^{4} \cdot$ Muthupandian Saravanan $^{5}$ (1)
}

Published online: 21 January 2020

(C) Springer Science+Business Media, LLC, part of Springer Nature 2020

\section{Correction to: Journal of Cluster Science https://doi.org/10.1007/s10876-019-01732-3}

In the original version of the article the acknowledgement section was unfortunately missed. The acknowledgement is published in this erratum article.

Acknowledgement The authors acknowledge the financial assistance from Kalasalingam University through the Summer Students Visiting Internship Programme (SSVIP2016) to undertake part the study and we highly thank Dr.
K. Selvaraj and appreciate the high-throughput instrumentation assistance for SEM, FTIR, EDS, and XRD of the Sir CV Raman-KS Krishnan International Research Center, Kalasalingam University, Krishnankoil, Tamilnadu. We also acknowledge K.S.Rangasamy College of Technology, Tiruchengode for supporting this research.

Publisher's Note Springer Nature remains neutral with regard to jurisdictional claims in published maps and institutional affiliations.
The original article can be found online at https:// doi.org/10.1007/s10876-019-01732-3.

Arulvel Ramaswamy

arulvelbabooo@gmail.com

$\triangle$ Muthupandian Saravanan bioinfosaran@gmail.com;

saravanan.muthupandian@mu.edu.et

1 Department of Biotechnology, K.S.Rangasamy College of Technology, Namakkal (District), Tiruchengode, Tamil Nadu 637 215, India

2 Department of Biotechnology, Saveetha School of Engineering, Saveetha Institute of Medical and Technical Sciences, Saveetha Nagar, Chennai, Tamil Nadu 602105, India

3 Department of Pharmaceutical Biotechnology, School of Pharmacy, Shahid Beheshti University of Medical Sciences, Tehran, Iran

4 Department of Food Science and Biotechnology, Sejong University, Seoul, Republic of Korea

5 Department of Medical Microbiology and Immunology, Division of Biomedical Science, School of Medicine, College of Health Sciences, Mekelle University, Mekelle, Ethiopia 